\title{
Trans-cuff leakage in bio-Bentall operation
}

\author{
Azumi Hamasaki ${ }^{1}$, Kozo Morita ${ }^{1}$, Masahiro Ikeda ${ }^{1}$, and Hiroshi Niinami ${ }^{1}$ \\ ${ }^{1}$ Tokyo Women's Medical University
}

October 6, 2021

\begin{abstract}
We have reported a case of trans-cuff leakage that occurred in a composite graft of bio-Bentall operation. The leakage resolved several months after surgery, similar to the trans-cuff leakage seen in simple aortic valve replacement. We have proposed hypotheses on the mechanism of trans-cuff leakage during a bio-Bentall operation and suggested ways to prevent it from occurring.
\end{abstract}

Title: Trans-cuff leakage in bio-Bentall operation

Running title: Trans-cuff leakage in bio-Bentall operation

Authors: Azumi Hamasaki, MD, PhD, Kozo Morita, MD, Masahiro Ikeda, MD, Hiroshi Niinami, MD, PhD

Institution: Department of Cardiovascular Surgery, Tokyo Women's Medical University, 8-1 Kawada-cho, Shinjuku 162-8666, Japan

Funding: none declared.

Corresponding author: Azumi Hamasaki

Department of Cardiovascular Surgery, Tokyo Women's Medical University, 8-1 Kawada-cho, Shinjuku 1628666, Japan

Tel.: +81-3-3353-8111, Fax: +81-3-3356-0441

E-mail:hamasaki-ths@umin.ac.jp

Abstract

We have reported a case of trans-cuff leakage that occurred in a composite graft of bio-Bentall operation. The leakage resolved several months after surgery, similar to the trans-cuff leakage seen in simple aortic valve replacement. We have proposed hypotheses on the mechanism of trans-cuff leakage during a bio-Bentall operation and suggested ways to prevent it from occurring.

Keywords: trans-cuff leakage, bioprosthesis, Bentall operation

Introduction

Aortic root replacement is a widely performed operation for diseased aortic root involving aortic valve insufficiency and sinus of Valsalva dilatation. Valve-sparing aortic root replacement is recommended for younger patients, while aortic root replacement using a bioprosthetic valve [1], so called 'bio-Bentall operation' is still the standard procedure for elder patients. Recently, with the improvement in the durability of bioprostheses, the indications for bio-Bentall operation have expanded.

A composite graft combined with a mechanical valve and a tube graft is commercially available; however, a composite graft of bio-Bentall operation needs to be hand sewn. The INSPIRIS RESILIA Aortic Valve 
(Edwards Lifesciences, Irvine, CA, USA) is a newly developed bioprosthesis, which is designed based on the Carpentier Edwards PERIMOUNT (CEP) Magna Ease aortic bioprosthesis (Edwards Lifesciences). This series of bioprostheses has a long history of excellent haemodynamics, but can cause trans-cuff leakage from the stent post due to its structural features. Trans-cuff leakage during aortic valve replacement (AVR) has been previously reported $[2,3]$, but none have reported trans-cuff leakage in composite grafts of bio-Bentall operation.

\section{Case Report}

A 68-year-old man with a dilated sinus of Valsalva and moderate aortic regurgitation underwent bio-Bentall operation. The sinus of Valsalva was $49 \mathrm{~mm}$ in diameter and the sino-tubular junction was $40 \mathrm{~mm}$ in diameter. The composite graft was prepared by hand sewing a 27-mm INSPIRIS RESILIA valve into a 30-mm Gelweave Valsalva (Vascutek Ltd., Inchinnan, UK) with a running 2-0 braided polyester suture. More specifically, the bioprosthesis was placed on top of the collar under the skirt of the graft. The composite graft was implanted supra-annularly with 2-0 pledgetted braided polyester non-everting mattress sutures. The threads were not applied to the sewing-cuff of the bioprosthesis, but it was applied to the graft collar. Finally, the edge of the graft collar and remnant of the Valsalva wall were sutured with a running 4-0 polypropylene suture to reinforce and the proximal anastomosis was completed. Reimplantation of the coronary ostia and distal anastomosis were performed in the standard fashion. Intra-operative transesophageal echocardiography (TEE) showed mild leakage blowing vertically from the sewing-cuff just below the left-right stent post (Figure 1 and Video 1). We judged the leakage to be trans-cuff leakage, which occasionally occurs in this particular bioprosthesis, and proceeded to complete the operation. Trivial trans-cuff leakage was noted on transthoracic echocardiography (TTE) performed 11 days postoperatively, and the patient was discharged. Trans-cuff leakage disappeared completely on TTE performed 6 months postoperatively.

\section{Comment}

There has been a long-standing concern among surgeons whether to follow-up or reintervene when intraoperative TEE reveals suspected perivalvular leakage. Our perception has changed since learning that trans-cuff leakage occasionally occurs in this particular bioprosthesis and most eventually resolves within 3 months [3]. This knowledge has guided our current practice to avoid unnecessary intervention.

In simple AVR, trans-cuff leakage occurs from the left-right commissure due to the anatomical features of the aortic annulus [3]. The nadir of the non-coronary cusp is usually at the lowest level compared to the remaining cusps, and when the CEP Magna Ease aortic bioprosthesis is secured at the nadir of the noncoronary cusp and implanted obliquely, the stent post of the left-right commissure is placed at a higher level. As a result, the weak point of the sewing-cuff may be stretched at the left-right commissure.

As in the present case, it is unclear why trans-cuff leakage occurs when the bioprosthesis is fixed to an artificial graft. In our case, we sewed suture threads from the outside of the graft at the middle of the graft to fix the bioprosthesis. The sewing-cuff must be sewn out of sight because the bioprosthesis was sewn in the middle and not at the edge of the graft. As a result, the depth of the sutures was uneven. In addition to the anatomical cause of trans-cuff leakage, Hiraoka et al. pointed out that applying stitches to the edges of the sewing-cuff increases the risk of leakage [3]. Trans-cuff leakage may have occurred because the sewing-cuff was sewn shallowly near the stent post, and the weak point of the sewing-cuff was stretched.

The ideal way to prevent trans-cuff leakage in a hand sewn composite graft of bio-Bentall operation is to sew the sewing-cuff at a uniform depth. When the bioprosthesis is sutured at the end of the graft, as in the double sewing ring technique [4], the sewing-cuff of the bioprosthesis is clearly visible during the procedure and the sewing needle can be passed through at a uniform depth; thus, trans-cuff leakage can be prevented. We have used this technique in several recent bio-Bentall operations, and no trans-cuff leakages have been reported.

Acknowledgments

We would like to thank Editage (www.editage.com) for English language editing. 


\section{Conflict of interest}

The authors declare that they have no conflict of interest.

Patient consent statement

A written informed consent was obtained from the patient.

Ethics statement

The need for ethics approval was waived by the institutional review board.

Author contributions: KM, AH and MI performed the surgical procedure and perioperative management. AH collected the data, drafted and critically revised the manuscript. All authors approved the article. HN supervised the study.

\section{References}

1. Etz CD, Homann TM, Rane N, et al. Aortic root reconstruction with a bioprosthetic valved conduit: a consecutive series of 275 procedures. J Thorac Cardiovasc Surg 2007;133:1455-63.

2. Tokunaga S, Cho T, Izubuchi R, Masuda M. Cuff leakage, not paravalvular leakage, in the Carpentier Edwards PERIMOUNT Magna Ease aortic bioprosthesis. Interact Cardiovasc Thorac Surg 2015;21:796-7.

3. Hiraoka A, Hayashida A, Totsugawa T, et al. Transprosthetic cuff leakage of a bovine pericardial aortic bioprosthesis. Semin Thorac Cardiovasc Surg 2019;31:773-9.

4. Albertini A, Dell'Amore A, Zussa C, Lamarra M. Modified Bentall operation: the double sewing ring technique. Eur J Cardiothorac Surg 2007;32:804-6.

Legends

Figure 1

Intra-operative transesophageal echocardiography showing mild leakage from the left-right stent post and the regurgitation jet blowing vertically from the sewing-cuff.

Video 1

Intra-operative transesophageal echocardiography showing the regurgitation jet from the left-right stent post blowing vertically from the sewing-cuff. 


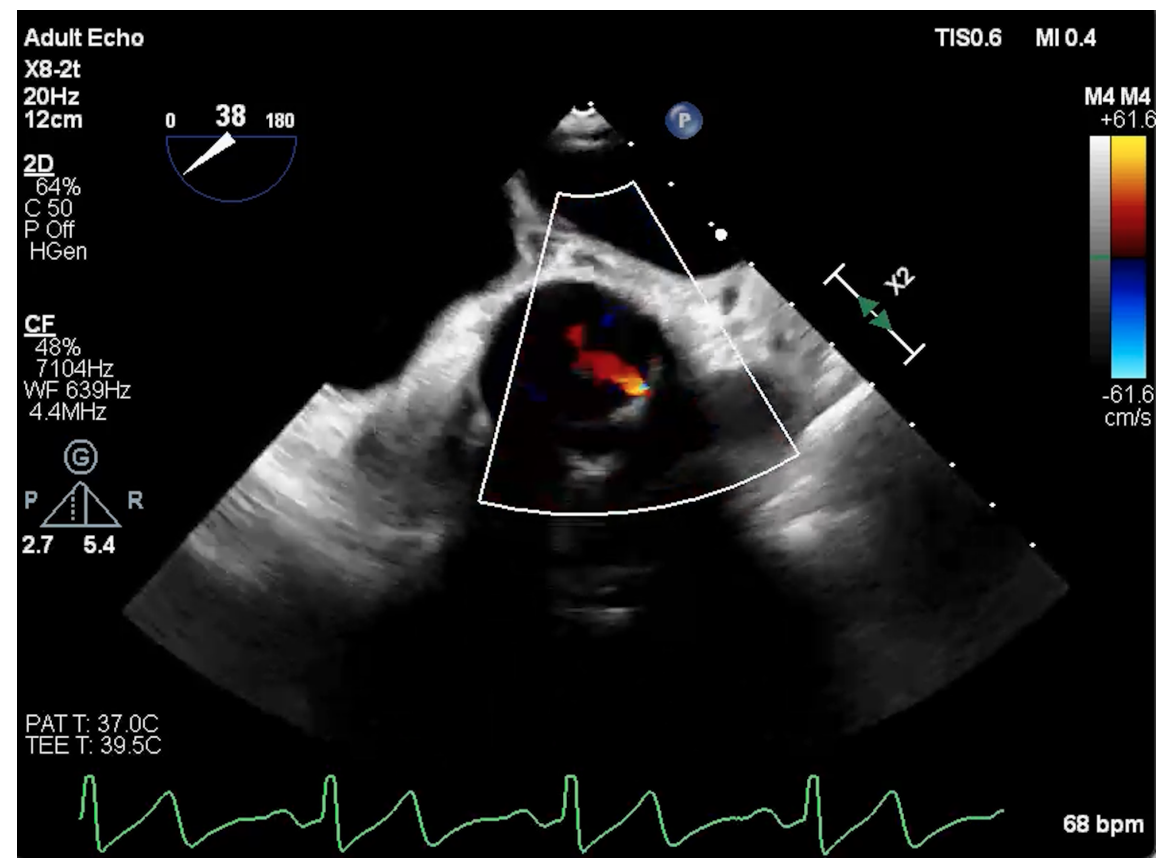

